\author{
¿Cómo citar este artículo? \\ Marín Castaño, J. P. y Padierna Cardona, J. C. (mayo-agosto, 2020). Representacio- \\ nes sociales sobre formación profesional en estudiantes de actividad física y \\ deporte en Medellín. Revista Virtual Universidad Católica del Norte, (60), 251-270. \\ https://www.doi.org/10.35575/rvucn.n60a13
}

\title{
Representaciones sociales sobre formación profesional en estudiantes de actividad física y deporte en Medellín
}

\section{Social representations about professional training in physical activity and} sport students in Medellin

\section{Juan Paulo Marín Castaño}

Candidato a doctor en Ciencias de la Educación Politécnico Colombiano Jaime Isaza Cadavid Medellín, Colombia juanmarin@elpoli.edu.co Orcid: https://orcid.org/0000-0001-9357-7981

\author{
Juan Carlos Padierna Cardona \\ Doctor en Educación \\ Universidad de Antioquia \\ Medellín, Colombia \\ juan.padierna@udea.edu.co \\ Orcid: https://orcid.org/0000-0001-8409-9914
}

Recibido: 1 de noviembre de 2019 Evaluado: 17 de marzo de 2020 Aprobado: 17 de abril de 2020 Tipo de artículo: Investigación Científica y Tecnológica

\section{Resumen}

Las representaciones sociales en el contexto de la formación posibilitan discernir la condición histórica, social y subjetiva de esta realidad, ubicando al actor educativo como un sujeto dinámico. El objetivo de esta investigación fue establecer las representaciones sociales sobre la formación profesional que tienen los estudiantes de actividad física y deporte en Medellín. Se empleó un diseño multi-metodológico anidado, que dio cuenta de la incorporación de métodos cuantitativos y cualitativos. La recolección de información se realizó a través de un cuestionario modificado y la técnica de redes semánticas naturales. Los resultados señalaron que la formación profesional para los estudiantes universitarios, no solo da cuenta de componentes educativos y disciplinares, sino que, además, se vincula con elementos sociales, éticos, transformacionales, de beneficio físico y de relación con el otro, canalizados desde elementos valóricos. El estudio concluye que el componente valórico, que a diferencia de lo educativo y lo disciplinar no aparece en los currículos establecidos, o por lo menos no de forma explícita, se convierte en un aporte conceptual relevante. Además, esta investigación hace un llamado a las instituciones a incluir en sus planes de estudio elementos de materia relacional, en donde los valores son un pilar fundamental.

Palabras clave: Actividad Física; Deporte; Estudiante universitario; Formación Profesional; Representaciones sociales. 


\section{Abstract}

Social representations in the context of formation makes possible discern the historical, social and subjective condition of this reality, locating the educational actor as a dynamic subject. The objective of this investigation was to establish social representations about professional formation that have the students of physical activity and sports in Medellin. A nested multi-methodological design was used that realized the incorporation of qualitative and quantitative methods. The collection of information was through a modified questionnaire and the technique of natural semantic networks. The results indicated that the professional formation to the university students, is not only for educational and disciplinary components, but also is linked with social, ethical, transformational, of physical benefit, and in relation to the other elements, grouped into valuable elements. This study concludes that the value component, which unlike educational a disciplinary, doesn't appear in the established curricula, or at least not explicitly, becomes a relevant conceptual contribution. Also, this investigation calls for institutions to include in their study plans relational matter elements, where values are a fundamental pillar.

Palabras clave: Physical Activity; Sport; University student; Professional formation; Social representations.

\section{| Introducción}

El espacio que se establece en el mundo y que posibilita ser el centro de las vivencias cotidianas, está diseñado de acuerdo con las palabras que describen el conocimiento, las creencias, percepciones y demás elementos de ese mundo; lo que según Saussure (1945) se denomina "el lenguaje como hecho social" (p. 34). De acuerdo con esto, plantea Pinker (2007) que es una cuestión semántica, dado que se "trata de la relación de las palabras con el pensamiento, pero también se ocupa de las palabras con otros asuntos humanos" (p. 17); es decir, con la manera en que las personas interpretan los significados de las palabras y se hacen una representación de la realidad.

La formación profesional se acoge a los planteamientos de Bunk (1994), en la que además de las competencias profesionales y genéricas se abarca intrínsecamente otro tipo de competencias, como las metodológicas, sociales y participativas, generando estas a su vez habilidades, movilidad y empleabilidad para los futuros profesionales. En este sentido, una acción de formación pedagógica es justificada si va dirigida a un objetivo, si relaciona la teoría y la práctica en un mismo entorno, si está planificada de manera constante, responsable, y si es medible y auto-evaluable.

De acuerdo con Rebelo (2016) diversos estudios han demostrado que cada vez más el entrenador tiene una función relevante en la formación de otros, lo que exige responsabilidades y conocimientos propios de la pedagogía. Esto, porque los estudiantes de educación superior de actividad física y deporte pueden ser percibidos como educadores en los diferentes contextos en los que tienen posibilidades de actuación, siendo fundamental que se formen conscientes de su función y actuación profesional, de acuerdo a las exigencias que le requiera el individuo o el grupo hacia al cual se enfoca, y teniendo en cuenta su responsabilidad social.

Las representaciones sociales (en adelante RS), por su parte, y según Moscovici (1961) son "entidades casi tangibles. Circulan, se cruzan y se cristalizan en nuestro universo cotidiano a 
través de una palabra, un gesto, un encuentro, éstas se constituyen en una sustancia simbólica que al mismo tiempo permea las prácticas, míticas o científicas" (p. 27).

Las representaciones aluden a la dialéctica entre lo individual y lo colectivo. Durkheim (1986) privilegió las representaciones colectivas. A su juicio la consciencia colectiva trasciende a los individuos, de este modo lo social es una construcción permanente entre la realidad y la representación. El concepto representación social fue instaurado por Moscovici (1961), considerando que esta es una forma específica de conocimiento, cuyo deseo es la producción de relaciones y prácticas entre los individuos. Weisz (2017), por su lado, manifiesta que la RS es un proceso cognitivo y social de sentidos y significados, elaborados culturalmente por el sujeto y sus correlaciones, lo que lleva a que el objeto se precise desde los dispositivos grupales.

El conocimiento de las RS que emergen en los diferentes contextos y grupos poblacionales conlleva esencialmente a la reflexión sobre las situaciones de la vida cotidiana, a la toma de decisiones que promueven la transformación de realidades concretas, en búsqueda de acciones de cambio y mejoramiento. Las RS no quedan atadas únicamente al aspecto cognitivo, sino que establecen realidades y relaciones intersubjetivas desde el entender del sentido común de las experiencias de la vida cotidiana.

En este sentido, se entienden las RS como el conocimiento del sentido común, en el que los sujetos se sitúan al interior de lo que conocen. Según Mora (2002) las RS tienen dos caras, una figurativa y otra simbólica, permitiendo designar a toda figura un sentido y a todo sentido una figura. El estudio del sentido común es relevante para entender y explicar las relaciones de intercambio social. Las RS tienen al tiempo una naturaleza estable y dinámica, son a la vez sistemas y procedimientos, concepciones sociales establecidas y constituyentes, fenómenos históricos y culturales; lo que supone que son cambiantes y modificables, llevando a que las personas se constituyan así mismas en relación con los demás y con el mundo (Banchs, 2000). En esta misma línea Rateau y Lo Monaco (2013) plantean que las RS se presentan como diversos "elementos cognitivos" (p. 21) sobre un objeto social que cumple cuatro características principales: organización o estructura (relación entre elementos), compartido por el grupo social, fabricado conjuntamente en ejercicios de comunicación, y su finalidad de ser socialmente útil.

De esta forma, los objetos de conocimiento, las relaciones entre los sujetos y el entorno de interpretación y acción se constituyen como uno de los intereses epistemológicos principales para las RS; además, ayudan en la conexión entre sujeto y objeto, entre saber y hacer, entre cognición y acción (Rubira-García y Puebla-Martínez, 2018).

Las RS según Navarro y Restrepo (2013) son “el resultado de la relación entre un objeto de representación y un sujeto que lo representa dentro de un marco histórico y cultural de referencia" (p. 2). En este sentido, las RS permiten identificar y comprender el pensamiento que se elabora socialmente, siendo útiles y empleadas como guías para la acción y la práctica, posibilitando la interacción y comunicación de personas que hacen parte de un grupo, e incluso permiten la interrelación con personas de otros grupos; es decir, las RS establecen y elaboran la singularidad de grupos sociales. Arnold (2001) plantea que las RS “constituyen sistemas de referencia que vuelven lógico y coherente el mundo para los sujetos, organizando las explicaciones sobre los hechos y las relaciones que existen entre ellos" (p. 300). Las RS no muestran exclusivamente lo que el sujeto exterioriza, pueden entenderse más bien como una elaboración de significados sobre el objeto que es representado; muestra ideas y configuraciones de los 
grupos sociales.

En este sentido, Martinic (2006) asigna tres componentes centrales a las RS: primero, es el contenido informacional, el cual evidencia las manifestaciones cognitivas y conceptuales que tiene la representación sobre la realidad estudiada; el segundo, es la colocación interna que orienta las categorías y sus relaciones, lo que muestra la configuración de la representación; y en tercer lugar, la dimensión ética - normativa que evalúa las significaciones y relaciones que la componen. De este modo, las RS evidencian la configuración y el sentido del objeto que es representado, estableciendo realidades y experiencias cercanas a los sujetos.

En el contexto de la formación profesional, las RS han sido resaltadas por su utilidad ya que dan luces para la comprensión de sus objetos. Alasino (2011) plantea que las RS son pertinentes para la construcción de investigaciones sólidas y ayudan a desentrañar las RS construidas por los actores de las comunidades educativas, interpretando el sentido y ayudando a su comprensión. Al respecto Materán (2008) plantea que el modo en que las RS participan en las prácticas educativas, instauran un objeto oportuno para la investigación, intervienen en la constitución de saberes y vinculan, además, el contexto y los factores sociales que son influyentes en los procesos de enseñanza y aprendizaje, debido a que la formación acoge procesos y productos establecidos culturalmente.

Las RS en el campo de la formación posibilitan discernir la condición histórica, social y subjetiva de esta realidad, ubicando al actor educativo como un sujeto dinámico, cuya función es dar forma a lo que proviene de su exterior. Las RS hacen frente a las posiciones de reproducción y deterministas que han negado la visión simbólica y subjetiva de la realidad en la educación. Además, las RS permiten una apertura de diferentes perspectivas sociales, antropológicas, históricas o cognitivas, y la utilización de diferentes métodos y tipos de acercamiento conceptual que favorece su utilización (Mireles, 2011). Por su parte, Lacolla (2005) plantea que los estudiantes son sujetos que llevan consigo realidades del contexto social y cultural al que pertenecen, posibilitando identificar las características que se manifiestan en el proceso de formación y en el aprendizaje de conceptos dados en el campo académico. Por tanto, las RS que tienen los estudiantes sobre su formación se manifiestan de acuerdo a la experiencia y a la relación dada entre el sujeto (estudiante) y el objeto (formación) en un momento histórico específico.

Al respecto, López, Correa, y Rojas (2017) mencionan que si se conocieran las RS y por tanto las subjetividades de los diversos actores educativos, se posibilitaría identificar los significados que los actores tienen sobre un objeto, lo que a la vez, serviría como guía para tomar posición y actuar sobre el objeto representado. De igual forma, Noreña et al. (2016) plantean que la relación de la formación profesional con las RS permite identificar las apreciaciones que tiene la comunidad sobre los elementos relacionados con los procesos formativos.

Las RS tienen gran relación con las teorías implícitas, las cuales se convierten en la formulación de dos modelos teóricos del sentido común (Castorina, Barreiro y Toscano, 2005). El estudio del sentido común plantea rasgos de las teorías implícitas; además, dan sentido a las experiencias del individuo en la vida cotidiana, a la exigencia de tomar decisiones para la acción. Las representaciones producidas de este modo tienen una función pragmática, más que epistemológica, lo que constituye junto a su carácter implícito el motivo principal de su resistencia a ser modificadas. 
El objeto de estudio es la formación profesional, la cual según Bunk (1994) tiene en cuenta elementos profesionales, metodológicos y sociales. Es necesario una vinculación profunda de teoría y práctica; además, el autor propone un modelo de formación profesional que incluye métodos reactivos y activos: en el primer caso el docente procede activamente y el alumno de manera pasiva; y en el segundo, inversamente, detallando además que este segundo método es imprescindible para la transmisión de las competencias a los estudiantes, dado que no se aprende a proceder por medio de la instrucción, sino por la propia acción.

Por su parte, Tejada y Navío (2005), al abordar la función de la formación en la gestión de competencias profesionales, destacan dos dimensiones elementales: la personal y la social, enfatizando en la función de gestión y progreso de las competencias profesionales de la formación. Los autores plantean que la formación profesional no puede reducirse a la aplicación de teorías de enseñanza, sino más bien al desarrollo de la acción de estas teorías, que incentive el terreno de la experiencia. Esto invita a que la formación profesional transforme los propios modelos, complementados con la experiencia y fomento de las capacidades propias de cada estudiante.

En este sentido, V. González y R. M. González (2008), al analizar las reflexiones de egresados y docentes universitarios de Cuba y Bolivia sobre la importancia de la formación profesional y las capacidades genéricas y su avance, encontraron inicialmente que el interés por estos conceptos no aparece en el escenario universitario, sino en el escenario del trabajo en la década de 1870, permitiendo que las competencias profesionales acerquen la formación profesional al mundo laboral. Según las autoras, la percepción de las competencias profesionales está relacionada con la formación del estudiante universitario, ya que no solo se vincula con conocimientos y habilidades del desempeño específico, sino con la formación motivacional de recursos personales. Desde este punto surgen las competencias profesionales genéricas (conocimientos generales que debe adquirir un estudiante) y competencias profesionales específicas (relacionadas con el saber hacer, saber guiar, saber estar y saber ser, propias de la profesión).

No obstante, Barreto, Celis y Pinzón (2019) muestran que la desigualdad de oportunidades para la población joven ha sido una particularidad de la economía colombiana; sumado esto a que las instituciones educativas no han equilibrado dichas desigualdades, debido a la diversidad de ofertas y programas académicos, lo que ha tenido como resultado el aumento de la cobertura, pero no la oportunidad de alcanzar una educación de calidad que posibilite competitividad laboral para los profesionales en formación.

Al respecto, Gorodokin (2006) expone cómo los procesos formativos y las ideas que se instauran en los estudiantes en formación, están atravesados por las acciones de las instituciones y, además, establecidos por las percepciones de los estudiantes y los contextos en los que interactúa con los otros, por lo que la subjetividad se convierte en un elemento fundamental de los procesos de formación y del futuro quehacer profesional. En este sentido, plantea Jiménez (2011) que "cuando se habla de subjetividad se ha de reconocer ésta como la posibilidad de indagación sobre el modo cómo el ser humano se convierte a sí mismo en sujeto" (p. 52).

Por todo ello, esta investigación apuntó a una concepción del estudiante de educación superior desde la subjetividad, de la vivencia del sujeto y de las relaciones que se generan con los otros en contextos diferentes, con el fin de que se tenga en cuenta la posición que el estudiante tiene frente a las situaciones que giran a su alrededor y la manera como se 
reorganiza a partir de estas.

Por otro lado, en la búsqueda teórica se han develado ausencias en publicaciones referidas a la relación de las RS y la formación profesional, permitiendo iniciar la indagación del valor de la subjetividad en estudiantes de educación superior; por lo que la teoría de las RS se convierte en una posibilidad de conocer dichos objetos y además de originar discusiones y cuestiones producidas desde otros enfoques, que han estado presentes en el estudio de la formación profesional.

También, se ha constatado, en los rastreos realizados, que la voz del estudiante de actividad física y deporte no se ha considerado adecuadamente, y ha sido una cuestión circunstancial que obedece a cuestiones administrativas, no a una renovación activa del currículo. Por el contrario, las disposiciones institucionales sí se evidencian, transforman y plantean por quienes, desde su función, asumen la tarea de mostrar dicha realidad.

Esto lleva a pensar que el estudiante en proceso de formación está vinculado a lineamientos y posturas institucionales, y su intervención como miembro de su campo disciplinar se limita a la reproducción de los discursos de otros, que en algunos casos hacen parte de la función social de la profesión, tales como los discursos culturales, políticos y académicos. Además, este puede estar expuesto a ideologías y poderes económicos que le afecten tener una postura crítica y reflexiva sobre las problemáticas de inequidad y exclusión que se dan alrededor de su profesión, llevando a que su función se limité a una posición distante, lo cual lo vincula como un sujeto que reproduce ideologías sin sentido crítico.

Ante esto, investigar acerca de las características de los estudiantes de educación superior se convierte en un aspecto justificable y fundamental, buscando que las RS sobre formación profesional sean tenidas en cuenta por las instituciones de educación superior, específicamente en los diseños curriculares y en los objetos misionales de docencia, investigación y extensión.

\section{| Metodología}

Las RS no se enfocan en un desarrollo metodológico específico. La elección de la metodología está orientada por el sistema teórico que respalda la investigación. En este caso, se sustenta en el modelo teórico de las RS desde el enfoque estructural y además por las "consideraciones empíricas" del estudio (Abric, 2001, p. 27). El enfoque estructural propuesto por Abric (2001), reclama la utilización de métodos que, por un lado, identifiquen y hagan surgir los componentes de la representación, y por otro lado conozcan cómo se organizan esos elementos, identificando el núcleo central de la representación.

Siguiendo a Abric (2001) "el triple objetivo implica una aproximación multi-metodológica de las representaciones, organizada en tres tiempos sucesivos" (p. 54), lo que posibilitará un acercamiento con la realidad del individuo o grupo indagado:

1) La identificación del contenido de la representación.

2) El estudio de las relaciones entre elementos, su importancia relativa y su jerarquía.

3) La determinación y el control del núcleo central. 


\section{Tipo de estudio}

Dadas las características del estudio y siguiendo la propuesta de Polit y Hungler (2005) se utilizó un diseño multi-método integrado-anidado, en el cual se incluyeron métodos cuantitativos y cualitativos; lo anidado hace referencia a la incorporación de un diseño al interior de otro. El diseño descriptivo (cuantitativo) se elaboró a partir de valores propios de las redes semánticas naturales (E. González, 2019; Valdez, 1998), y el procesamiento de los valores se realizó a partir de la utilización de las funciones de tablas dinámicas de Excel para determinar el valor J, valor M, valor FMG y valor PQ (estos valores se explican en las técnicas e instrumentos). Para el análisis de los casos múltiples (cualitativo) se utilizó el agrupamiento por categorías, de acuerdo a la codificación axial (Strauss y Corbin, 2002).

Se elaboró una ruta de trabajo que dio cuenta de la comprensión de la realidad de los estudiantes sobre formación profesional; dicha comprensión se realizó en el contexto universitario. Se privilegió la voz de los estudiantes, sus percepciones, subjetividades, experiencias, vivencias, significados, los cuales están dados por su conocimiento y limitación de su apreciación, a partir de palabras y argumentos que muestran las principales categorías y redes semánticas naturales, las cuales ilustran su realidad.

Por las características cualitativas del estudio se empleó el diseño de estudios de caso múltiples (Chaverra, Gaviria y González, 2019); por tanto, el análisis se abordó teniendo en cuanta las representaciones dadas por los estudiantes de cada institución, y luego se presentó una síntesis que agrupa los encuentros y desencuentros de los casos en general.

Desde la perspectiva cuantitativa se empleó un diseño no experimental, pues no se manipularon variables independientes. En este estudio no se realizó intervención alguna y lo que se buscó fue caracterizar el fenómeno de estudio; específicamente se utilizó un diseño descriptivo, el cual tuvo el propósito de "observar, describir y documentar aspectos de una situación que ocurre de manera natural” (Polit y Hungler, 2005, pp. 190-191).

\section{Población y muestra}

El objeto estudiado (caso) fueron las RS sobre formación profesional, a partir del estudio de 3 casos que corresponde a la totalidad de instituciones de educación superior, que se encargan de la formación de estos profesionales en la ciudad de Medellín. La población de estudio fue de 2019 estudiantes.

La muestra para la recolección de información, su análisis descriptivo y de redes semánticas naturales se elaboró a partir de un muestreo probabilístico estratificado (Grisales, 2001), teniendo en cuenta los criterios de confiabilidad (95\%), error (5\%), probabilidad de ocurrencia $(P=50 \%)$, probabilidad de no ocurrencia $(Q=50 \%)$, población $(N=2019)$ y muestra final $(n=306)$. La manera como se seleccionó la muestra se presenta en la tabla 1 de los resultados.

La selección de los sujetos se hizo a partir de un procedimiento aleatorio simple. Se escogieron aleatoriamente un grupo por semestre y los estudiantes a indagar. Para la escogencia de los grupos y los estudiantes se utilizó una tabla de números aleatorios (Polit y Hungler, 2005). El trabajo de campo se realizó en el segundo semestre académico del año 2018. 


\section{Técnicas e instrumentos}

Se utilizó una encuesta (cuestionario) modificada, a partir de la propuesta de E. González (2019), dirigida a la muestra representativa de estudiantes de las tres instituciones indagadas, con preguntas referidas al perfil social y académico, y apreciaciones sobre la formación profesional. También, en la muestra representativa se hizo uso de la técnica de redes semánticas naturales (guía de palabras estímulo), las cuales son catalogadas como un procedimiento de carácter mixto, ya que ofrecen la posibilidad de ser analizadas desde un punto de vista cuantitativo o cualitativo (Hinojosa, 2008).

Los principales valores de las redes semánticas naturales, de acuerdo con Valdez (1998), son los siguientes:

Valor J: muestra la riqueza semántica de la red; es decir, a mayor cantidad de palabras definidoras sobre un objeto social mayor es su riqueza.

Valor M: es un indicador del peso semántico y se logra al multiplicar la frecuencia de aparición con la jerarquía dada a cada una de las palabras generadas por los sujetos.

Conjunto SAM: establece el núcleo central de la red semántica y por ende la estructura central de la RS.

Valor FMG: indica en porcentajes la distancia semántica entre las distintas palabras que conforman el conjunto SAM. Se obtiene partiendo de la palabra definidora con el valor M más alto de la red, que equivale al $100 \%$.

Para esta investigación se tomó como referencia para la determinación del conjunto SAM o la representación hegemónica, la propuesta de punto de quiebre (en adelante PQ), para lo cual se escogieron aquellas palabras que tuvieron una distancia semántica igual o superior al 5\%, con respecto a la palabra definidora principal (E. González, 2019). El procesamiento de la información se realizó en la herramienta de cálculo Excel.

\section{| Resultados}

El cálculo inicial de la muestra, indicó que era necesario trabajar con 323 sujetos, lo que superó el $10 \%$ de la población. Por tanto, se ajustó la muestra y se obtuvo un valor de 278 sujetos. Con el fin de prever complicaciones se realizó un sobre muestreo del 10\%, que equivale a 28 sujetos, por lo que la muestra final fue de 306 estudiantes.

Cada estrato de la población se refiere a cada una de las instituciones. En cada caso se tomó proporcionalmente una muestra, de acuerdo al porcentaje de la población: Institución 1 (13.1\%), Institución 2 (54.5\%) e Institución 3 (32.4\%). 
Tabla 1

Datos de la población y el muestreo

\begin{tabular}{cccccc}
\hline Instituciones & $\mathrm{N}$ & $\%$ & $\mathrm{n}$ & $\hat{\mathrm{N}}$ & $\mathrm{n}+10 \%$ \\
\hline Institución 1 & 264 & $13.1 \%$ & 42 & 369 & 40 \\
Institución 2 & 1100 & $54.5 \%$ & 176 & 152 & 167 \\
Institución 3 & 655 & $32.4 \%$ & 105 & 90 & 99 \\
Total & 2019 & $100 \%$ & 323 & 278 & 306 \\
\hline
\end{tabular}

Nota: Los datos de la población proceden de las oficinas de las direcciones académicas o de programa de las instituciones (2018). Los cálculos muéstrales son realizados por los autores.

La descripción de los hallazgos referidos a las diferentes RS se hace a partir del enfoque estructural, en el que es necesario dar cuenta del contenido y de la estructura de los objetos sociales indagados. Los resultados de las redes semánticas naturales, en el componente cuantitativo, se presentan con los diferentes valores semánticos, expresados en datos numéricos; y desde el componente cualitativo, a partir de la interpretación y agrupación por categorías de las diferentes palabras. Además, se presentan las discrepancias y semejanzas que se dan en cada una de las instituciones estudiadas.

La red semántica general sobre formación profesional fue definida con un total de 304 palabras (Valor J) que conforman la matriz total, lo que dio cuenta de una gran riqueza semántica. La representación total hegemónica, es decir, las principales palabras que determinan esta representación, estuvo conformada por 20 palabras (ver tabla 2) las cuales se escogen a partir del $\mathrm{PQ}$, o sea aquellas palabras que tienen un valor FMG igual o mayor a $5 \%$. La palabra principal fue aprendizaje (100\%), seguida de conocimiento (84,78\%), disciplina $(65,87 \%)$, salud $(46,54 \%)$, educación (46,10\%) y responsabilidad (34,66\%), las cuales en conjunto con las otras palabras del conjunto SAM proponen una serie de categorías o multi-significados que agrupan estas representaciones. 
Tabla 2

Representación social acerca de la formación de la población general

\begin{tabular}{lcccc}
\hline \multicolumn{1}{c}{$\begin{array}{c}\text { Palabra Estimulo: } \\
\text { Formación }\end{array}$} & Orden & Frecuencia & $\begin{array}{c}\text { M(Peso } \\
\text { semántico) }\end{array}$ & $\begin{array}{c}\text { FMG (Distancia } \\
\text { semántica) }\end{array}$ \\
\hline Aprendizaje & 243 & 73 & 17739 & 100,00 \\
Conocimiento & 235 & 64 & 15040 & 84,78 \\
Disciplina & 205 & 57 & 11685 & 65,87 \\
Salud & 172 & 48 & 8256 & 46,54 \\
Educación & 174 & 47 & 8178 & 46,10 \\
Responsabilidad & 143 & 43 & 6149 & 34,66 \\
Formación & 112 & 36 & 4032 & 22,73 \\
Entrenamiento & 104 & 35 & 3640 & 20,52 \\
Investigación & 105 & 34 & 3570 & 20,13 \\
Planificación & 86 & 28 & 2408 & 13,57 \\
Respeto & 85 & 26 & 2210 & 12,46 \\
Integral & 80 & 27 & 2160 & 12,18 \\
Enseñanza & 75 & 26 & 1950 & 10,99 \\
Preparación & 73 & 25 & 1825 & 10,29 \\
Deporte & 71 & 25 & 1775 & 10,01 \\
Práctica & 67 & 25 & 1675 & 9,44 \\
Compromiso & 71 & 23 & 1633 & 9,21 \\
Pasión & 78 & 20 & 1560 & 8,79 \\
Dedicación & 70 & 20 & 1400 & 7,89 \\
Bienestar & 49 & 18 & 882 & 4,97 \\
Valor J=304 & & & & \\
\hline
\end{tabular}

\section{Nota: Elaboración propia.}

Las categorías que agruparon las diferentes palabras de las RS, dadas por los estudiantes, fueron (ver tabla 3): educativo $(M=56.697)$, valórico $(M=30.720)$ y disciplinar $(M=24.546)$, lo que muestra que el componente educativo fue lo más relevante. Su representación social principal está en torno a la formación, y de esta categoría la palabra definidora principal es el aprendizaje (100\%). En pocas palabras, los estudiantes dan gran relevancia a los diferentes conocimientos y saberes que adquieren en su proceso formativo.

En segunda instancia, la categoría que recoge un gran número de palabras definidoras y sus respectivos pesos semánticos es la categoría valórica, es decir, representaciones que dan cuenta de valores y principios que son fundamentales en su formación, entre los cuales se destacan la disciplina (65,87\%), como el elemento principal; igualmente, aparecen palabras como: responsabilidad (34,66\%), respeto (12,46\%), integral (12,18\%), compromiso $(9,21 \%)$, pasión $(8,79 \%)$, entre otras. 
Finalmente emerge una categoría que da cuenta del campo particular del entrenador, descrita como disciplinar; es decir, aquellas designaciones que son propias de la formación de un entrenador deportivo o de actividad física. En esta categoría la palabra con mayor peso semántico es la salud (46,54\%), palabra que además denota que en el campo especifico, el entrenamiento y el rendimiento deportivo no es lo único que prima; también, aparecen palabras que se esperaban desde lo especifico de la formación de un entrenador, tales como: entrenamiento (20,52\%), planificación (13,57\%), preparación (10,29\%), deporte (10,01\%), entre otras.

\section{Tabla 3}

\section{Categorias que agrupan las palabras definidoras de la representación social de formación}

\begin{tabular}{cc}
\hline Categoria & Peso semántico \\
\hline Educativo & 56.697 \\
Valórico & 30.720 \\
Disciplinar & 24.546 \\
\hline
\end{tabular}

\section{Nota: Elaboración propia.}

En lo referido al análisis de cada una de las representaciones de los estudiantes por institución, se encontró que a mayor muestra indagada (n) (ver tabla 1) mayor número de palabras definidoras (Valor J). Para el caso de los estudiantes de la Institución 2 ( $n=167)$, la cual es de carácter público y con el programa denominado Profesional en Deporte, el valor J fue de 231 palabras; en la Institución 3 ( $n=99$ ), universidad privada y su programa Profesional en Actividad Física y Deporte, este valor fue de 150; y en la Institución 1 ( $n=40)$, universidad pública con el programa Profesional en Entrenamiento Deportivo, el valor J fue de tan solo 106.

No obstante, esto no corresponde con la RS hegemónica, pues para el caso de los estudiantes de la Institución 1, la representación principal o conjunto SAM estuvo conformada por 39 palabras, en la Institución 3 por 19 y en la Institución 2 por 18 palabras. Por tanto, los estudiantes de la Institución 1 tienen un mayor número de palabras que determinan la RS hegemónica sobre su formación profesional; para el caso de los estudiantes de las otras dos instituciones lo hegemónico es en cantidad de palabras muy similar (ver figuras 1, 2 y 3).

En el análisis categorial o por multi-significados, tanto los estudiantes de la Institución 2 y 3 , las tres categorías que emergieron en el análisis tuvieron el mismo orden de importancia que la representación general que agrupó la información de toda la muestra, incluso compartieron la misma palabra definidora principal que es Aprendizaje; y las dos palabras siguientes, aunque no en el mismo orden y peso semántico son conocimiento y disciplina, siendo entonces la disciplina, la principal palabra que en estos dos grupos de estudiantes designa lo valórico. Para el caso de lo disciplinar la palabra salud es la principal, al igual que en la representación general.

Para el caso de los estudiantes de la Institución 1, las tres categorías de análisis mencionadas, también dieron cuenta de toda la representación social sobre su formación, no obstante, el orden y pesos semánticos son diferentes. Estos estudiantes privilegiaron en su representación el componente disciplinar, seguido de lo valórico y, finalmente, lo educativo. Frente a lo disciplinar 
la palabra principal fue entrenamiento, en lo valórico la responsabilidad y en lo educativo el conocimiento.

En la figura 1 puede apreciarse la riqueza gramatical de la representación hegemónica de los estudiantes de la Institución 1 (J=39). Esto denota múltiples significados y posturas frente a su formación; es decir, en vez de privilegiar pocas designaciones con pesos muy altos, la formación para estos estudiantes apuesta por una red amplia de significados de su formación.

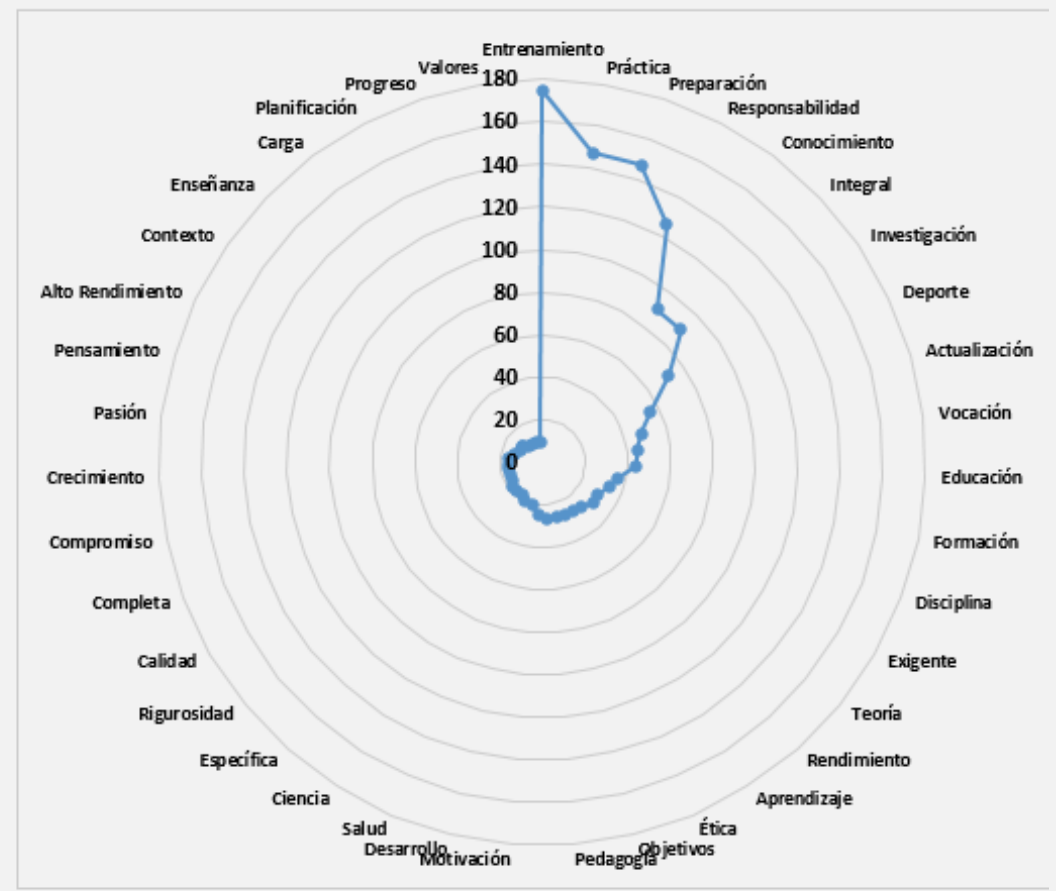

Figura 1. Conjunto SAM formación profesional: Institución 1. Elaboración propia. 
En las figuras 2 y 3 se aprecian las similitudes en las primeras palabras que conforman lo hegemónico de los estudiantes de la Institución 2 y los de la Institución 3, las cuales se resumen en: aprendizaje, conocimiento, disciplina, educación y salud. En este caso las RS sobre la formación son un punto de encuentro particular, pues son estudiantes de dos tipos de Instituciones: una pública (Institución 2), donde el énfasis está en lo deportivo; y otra privada (Institución 3) que enfatiza en la actividad física y salud. Por tanto, se esperarían representaciones más dispares, pues las RS se encuentran ancladas a las características de los grupos, lo que al parecer no se cumple en estos dos grupos de estudiantes.

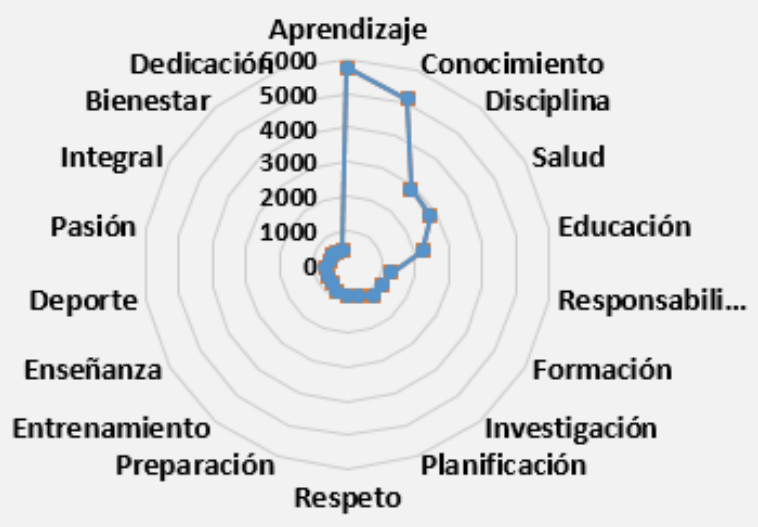

Figura 2. Conjunto SAM formación profesional: Institución 2. Elaboración propia. 


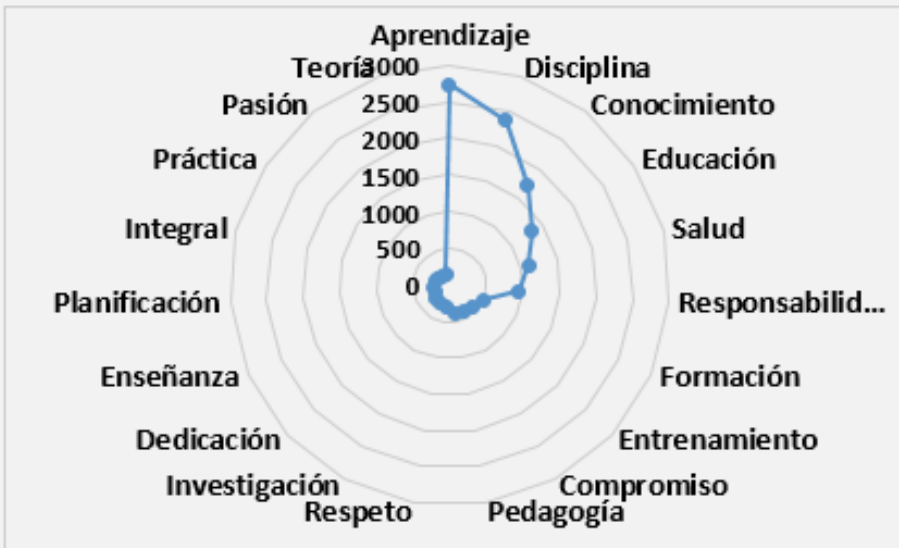

Figura 3. Conjunto SAM formación profesional: Institución 3. Elaboración propia.

\section{Discusión}

Uno de los aportes importantes de este trabajo, es que posibilitó el reconocimiento de las voces de los estudiantes indagados, su actitud respecto al objeto estudiado y la organización de su representación; elementos que tienen implicaciones prácticas y son de gran relevancia al momento de diseñar currículos y programas de estudio, que estén en armonía con las necesidades de los estudiantes. Al respecto, Valencia y Lizandra (2018) sugieren que los procesos de formación fomenten la novedad en los diseños curriculares, ligados a una formación que establezca un sentido crítico de las representaciones particulares que han fabricado los propios estudiantes.

De igual forma, este estudio presenta como elemento agregado, y de acuerdo a las orientaciones de E. González (2019), la identificación del punto de quiebre en las RS hegemónicas, planteando un valor igual o superior al $5 \%$ en la distancia semántica, para constituir la cantidad de palabras que conforman el núcleo central de una RS. Este valor se puede utilizar como principio en diversas investigaciones que utilicen en la recolección de información la técnica de redes semánticas naturales.

En cuanto al contenido de las representaciones encontradas, se puede plantear que la formación profesional se encuentra asociada principalmente con el aprendizaje (100\%) y el conocimiento (84,78\%), elementos propios del campo de la educación; del cual, también emergen palabras como investigación (20,13\%) y enseñanza (10,99\%).

A propósito de la formación de investigación en estudiantes de pregrado, Córdoba (2016) resalta que en Latinoamérica y específicamente en Colombia permanecen obstáculos para su desarrollo, vinculado esto a una tradición del "modelo napoleónico"(p. 35), complejo de cambiar 
tanto por las instituciones, los docentes y el Estado. En este sentido, se invita a partir de los elementos encontrados en este trabajo, generar propuestas para abordar la formación en investigación con elementos didácticos, educativos, organizativos e inversión de las instituciones.

Se hace necesario entonces, en la formación profesional, la integración de las competencias referidas con el aprendizaje y aquellas sobre los valores asociados al desempeño profesional. Siendo un reto para las universidades no solo diseñar currículos que potencien los dominios profesionales, que impliquen cambios en las dinámicas de enseñanza - aprendizaje y en las acciones de estudiantes y profesores, sino además de entender la formación como una conexión indispensable que responda a las exigencias de una sociedad globalizada, que lleve a los profesionales a ser autónomos, a gestionar incesantemente su conocimiento, comunicarse, trabajar en equipo, investigar y tener apertura para aprender en el transcurso de su vida.

A propósito, Gargallo (2016) menciona que es una exigencia de la sociedad actual, que los estudiantes sean estratégicos/autorregulados, capaces de aprender a aprender, diseñar, gestionar, controlar y optimizar sus procesos de aprendizaje y construcción del conocimiento; es decir, estudiantes con acción en su propia formación, en la que conciban el aprendizaje como un aspecto permanente a lo largo de la vida.

En el contexto universitario es clara la necesidad que tienen los estudiantes de aprender, un principio básico de la educación actual y una tarea esencial de la universidad, de modo que las dificultades que se le puedan presentar en el proceso de aprendizaje sean resueltas por ellos mismos y con el acompañamiento del docente, de manera que este vínculo consolide fundamentos teóricos, reflexión crítica, apropiación del conocimiento y habilidades necesarias de cada campo disciplinar.

En este sentido, las RS sobre formación profesional también acogen elementos disciplinares relacionados con la salud, el entrenamiento, la preparación, el deporte y la práctica, asuntos que concuerdan con el estudio de Caniuqueo et al. (2018), en tanto, al analizar las RS sobre educación física en estudiantes de esta disciplina en Chile, encontraron que el núcleo central estuvo representado principalmente por salud, deporte, motricidad y movimiento.

Así mismo, Cuadra-Martínez, Georgudis-Mendoza y Alfaro-Rivera (2012), al estudiar la RS de deporte y educación física en estudiantes con obesidad de la básica y la media en Atacama (Chile), hallaron que tanto el deporte como la educación física se definieron esencialmente desde una relación con la salud, el ejercicio, la diversión y el juego.

Además, la formación profesional incluye componentes de la esfera ética, tales como la responsabilidad, la disciplina, el respeto y la dedicación. Estos resultados coinciden con el estudio de E. González (2019), quien indagó las RS de formación inicial en estudiantes de educación física en Antioquia, en donde se destaca la alteridad como categoría transversal en las voces de estudiantes de educación física, pues consideran que en la función de un maestro está implícita la acción de trabajar y relacionarse con el otro.

Esto último también es reportado por los sujetos indagados en este estudio; es decir, en la condición de formarse como entrenador, el ser social y estar en función de otros, es un componente clave en su proceso de profesionalización, tal como lo establece Durkheim (1986), 
quien además indica que la condición de moralidad está dada por la búsqueda del bienestar de los otros, es decir, se logra en la función social del ser humano.

Así mismo, las universidades, según V. González y R. M. González (2008) deben “concebir la educación en valores como un eje transversal en la formación profesional, lo que implica lograr un aprendizaje profesional, ético y de compromiso social" (p. 204). En este sentido, la formación profesional debe tener en cuenta elementos para la vida en sociedad, para entender las relaciones comunitarias, de trabajo y actuar con un enfoque transformador, desde el vínculo que se genera entre la universidad, el trabajo y la ciudadanía.

Finalmente, se encontró un elemento esencial, y fue la determinación de la actitud de los estudiantes, que en el caso de este estudio fue bastante positiva, pues no se halló ninguna designación negativa, lo que mostró una posición y valoración favorable de los estudiantes respecto a su proceso de formación; asunto que coincide con el estudio de Noreña et al. (2016) sobre RS de la universidad pública en una región de Antioquia, en el que los estudiantes tuvieron una valoración entre buena y excelente de la calidad universitaria y por ende de su proceso de formación profesional.

\section{Conclusiones}

Las RS identificadas permiten postular que la formación profesional se encuentra distribuida en las categorías educativo, valórico y disciplinar; esta última, dadas las características de formación y proyección profesional de los estudiantes.

Con este estudio se entiende que la formación profesional está directamente relacionada con el campo de la educación, ya que emergen elementos vinculantes con el aprendizaje, el conocimiento, la investigación, la preparación y la enseñanza; es decir, la formación profesional se concibe como la encargada de educar y preparar a las personas para desenvolverse correctamente, no solo en un campo profesional especifico, sino también en las demás esferas de la vida.

Emergió como elemento clave la relación con el otro, situación que se consolida y ratifica desde el componente valórico; elemento que a diferencia de lo educativo y disciplinar no aparece en los currículos establecidos, o por lo menos no de forma explícita, lo que se convierte en un llamado a las instituciones a incorporar en sus planes de estudio elementos de materia relacional, en donde los valores parecen ser un pilar fundamental: responsabilidad, respeto, disciplina, compromiso, confianza, solidaridad, paciencia y amor; es decir, la formación profesional no debe centrarse exclusivamente en aspectos académicos, sino que debe cultivar los valores del ser humano, educar, dirigir y guiar hacia el conocimiento de manera consciente.

La formación profesional en el componente social se vincula con aspectos que tienen en cuenta la comunicación, el lenguaje, la interacción, la diversidad, la cultura, la sociedad, la participación, el contexto, el acompañamiento y el apoyo, permitiendo además que las personas vivencien y conozcan ámbitos de la vida que antes desconocían. Así mismo la formación profesional se convierte en una oportunidad de transformación social a partir del cambio que se da alrededor de cada estudiante, pues debe ofrecer herramientas de colaboración y trabajo cooperativo que lleven a la construcción de una sociedad más justa y equitativa. 
En el componente disciplinar, se puede plantear que la formación profesional está relacionada con la esfera de la salud, pues se vincula con el bienestar, la prevención, el cuerpo, el ejercicio, el entrenamiento físico, el deporte y la diversión; es decir, la formación profesional también debe beneficiar a las personas en su componente físico, emocional y social.

Por otro lado, se presentó como posibilidad y novedad para el procedimiento metodológico, específicamente en la determinación del punto de quiebre en el momento de identificar el núcleo central de una representación, desde la técnica de las redes semánticas naturales, el valor igual o mayor del 5\%, el cual diferencia los constituyentes hegemónicos y periféricos de una representación.

El proceso sobre el punto de quiebre mencionado se puede emplear como referente para determinar el contenido de un objeto representado, en el que prevalezcan los saberes, ideales y actitudes de un colectivo especifico. En este sentido, las RS además de ser una oportunidad para la generación de teoría sustantiva, es a la vez un componente metodológico para la elaboración de instrumentos de investigación.

Como líneas futuras y posibles trabajos de investigación en los que se emplee el diseño metodológico y el enfoque de las RS, utilizado en el presente estudio, estaría realizar investigaciones en otras áreas del campo del conocimiento de la educación física, tales como la administración deportiva, la actividad física para la promoción de la salud, la educación física escolar y la recreación, el ocio y el uso del tiempo libre, potenciando así el diseño metodológico empleado en el presente estudio.

\section{| Referencias}

Abric, J. C. (2001). Prácticas sociales y representaciones (1 ed.). México: Coyoacán.

Alasino, N. (2011). Alcances del concepto de representaciones sociales para la investigación en el campo de la educación. Revista Iberoamericana de Educación, 56(4), 1-11.

Arnold, R. (2001). Formación profesional. Nuevas tendencias y perspectivas (1 ed). Ginebra, Suiza: Organización Internacional del trabajor.

Banchs, M. (2000). Aproximaciones Procesuales y Estructurales al estudio de las Representaciones Sociales. Papers on Social Representations. 9, 3.1-3.15.

Barreto, D. A., Celis, C. G. y Pinzón, I. A. (septiembre-diciembre, 2019). Estudiantes universitarios que trabajan: subjetividad, construcción de sentido e in-satisfacción. Revista Virtual Universidad Católica del Norte, (58), 96-115. https://doi.org/10.35575/rvucn.n58a4

Bunk, G. (1994). La trasmisión de las competencias en la formación profesional y perfeccionamiento profesional de la RFA. Revista Europea, 1, 8-14. 
Caniuqueo, A., Hernández-Mosqueira, C., Troyano, A., Riquelme, D., Vargas, A., Vargas, R. y Fernandes, J. (2018). Representaciones sociales: el significado de la educación física para los estudiantes de esa disciplina. Revista Electrónica de Investigación Educativa, 20(1), 104-111. https://doi.org/10.24320/redie.2018.20.1.1497

Castorina, J., Barreiro, A. y Toscano, A. (2005). Dos Versiones del Sentido común: las teorías implícitas y las representaciones sociales. En J. A. Castorina (Coord.), Construcción conceptual y representaciones sociales El conocimiento de la sociedad (pp. 205-254). Buenos Aires, Argentina: Miño y Dávila.

Chaverra, B., Gaviria, D. y González, E. (enero, 2019). El estudio de caso como alternativa metodológica en la investigación en educación física, deporte y actividad física. Conceptualización y aplicación. Retos. Nuevas Tendencias En Educación Fisica, Deporte y Recreación, 35, 371-377.

Córdoba, M. E. (febrero-mayo 2016). Reflexión sobre la formación investigativa de los estudiantes de pregrado. Revista Virtual Universidad Católica Del Norte, (47), 20-37.

Cuadra-Martínez, D., Georgudis-Mendoza, C. N. y Alfaro-Rivera, R. A. (2012). Representación social de deporte y educación física en estudiantes con obesidad. Revista Latinoamericana de Ciencias Sociales, Niñez y Juventud, 10(2), 983-1001.

Durkheim, E. (1986). Las reglas del método sociológico (1 Ed.). México: Fondo De Cultura Ecónomica.

Gargallo, L. (2016). Métodos centrados en el aprendizaje y aprendizaje autorregulado en la sociedad del conocimiento. En M. A. Santos (Coord.), Sociedad del conocimiento: Aprendizaje e Innovación en la Universidad (pp. 115-136). Madrid, España: Biblioteca Nueva.

González, E. (2019). Representaciones sociales sobre la formación inicial y ser maestro en estudiantes de educación física del departamento de Antioquia (Tesis de Doctorado) Universidad de Antioquia, Medelllín, Colombia.

González, V. y González, R. M. (2008). Competencias genéricas y formación profesional: un análisis desde la docencia universitaria. Revista Iberoamericana Educación, 47, 185-209.

Gorodokin, I. C. (2006). La formación docente y su relación con la epistemología. Revista Iberoamericana de educación, 37(5), 1-10.

Grisales, H. (2001). Muestreo en estudios descriptivos. Medellín, Colombia: Facultad Nacional de Salud Pública - Universidad de Antioquia.

Hinojosa, G. (2008). EL tratamiento estadístico de las redes semánticas naturales. Revista internacional de Ciencias Sociales y Humanidades, 18(1), 133-154. 
Jiménez, J. R. (2011). La subjetividad del maestro en Colombia: la tensión entre la formación institucional y las prácticas de formación (1960 - 2002). Educación y Ciudad, (20), 48-58.

Lacolla, L. (julio-diciembre 2005). Representaciones sociales: una manera de entender las ideas de nuestros alumnos. Revista leRed: Revista Electrónica de La Red de Investigación Educativa, 1(3), 1-17.

López, L., Correa, L. y Rojas, M. (febrero-mayo 2017). Representaciones sociales: formación y uso de tecnologías de información y comunicación. Profesores de educación básica secundaria. Revista Virtual Universidad Católica Del Norte, (50), 256-276.

Martinic, S. (2006). El estudio de las representaciones sociales y el análisis estructural del discurso. En M. C. Cerón (Ed.), Metodologías de investigación social. Introducción a los oficios (pp. 299-320). Santiago, Chile: Lom Ediciones.

Materán, A. (julio-diciembre 2008). Las representaciones sociales: un referente teórico para la investigación educativa. Geoenseñanza, 13(2), 243-248.

Mireles, O. (enero-julio 2011). Representaciones sociales: debates y atributos para el estudio de la educación. Sinéctica, 36, 1-11.

Mora, M. (2002). La teoría de las representaciones sociales de Serge Moscovici. Athenea Digital, 2, 1-25. https://doi.org/10.5565/rev/athenead/v1n2.55

Moscovici, S. (1961). El Psicoanálisis, su imagen y su público. Buenos Aires, Argentina: Huemul.

Navarro, O. y Restrepo, D. (enero-junio, 2013). Representaciones Sociales: perspectivas teóricas y metodológicas. CES Psicología, 6(1), 1-4.

Noreña, M., Muñoz, S., Valencia, L., Córdoba, P., Orozco, J. y Guarín, G. (mayo-agosto 2016). Representaciones sociales sobre universidad pública de los habitantes entre los 15 y 24 años de los municipios de El Carmen de Viboral, La Ceja del Tambo y Rionegro. Revista Virtual Universidad Católica Del Norte, (48), 136-154.

Pinker, S. (2007). El mundo de las palabras. Una introducción a la naturaleza humana. Bercelona, España: Paidós Ibérica.

Polit, D. y Hungler, B. (2005). Investigación científica en ciencias de la salud: principios y métodos (6 ed.). México: McGraw-Hill.

Rateau, P. y Lo Monaco, G. (2013). La Teoría de las Representaciones Sociales: Orientaciones conceptuales, campos de aplicaciones y método. CES Psicología, 6(1), 22-42.

Rebelo, L. (2016). Estudio comparativo entre los entrenadores de élite de natación de Portugal. Concepciones y conocimiento pedagógico (Tesis de doctorado). Universidad de extremadura. Cáceres, España. 
Rubira-García, R. y Puebla-Martínez, B. (enero-abril, 2018). Representaciones sociales y comunicación: apuntes teóricos para un diálogo interdisciplinar inconcluso. Convergencia, 76, 147-167. https://doi.org/10.29101/crcs.v25i76.4590

Saussure, F. (1945). Curso de Linguística general (24 ed.). Buenos Aires, Argentina: Losada.

Strauss, A. y Corbin, J. (2002). Bases de la investigación cualitativa. Técnicas y procedimientos para desarrollar teoría fundamentada. Medellín, Colombia: Universidad de Antioquia.

Tejada, J. y Navío, A. (enero, 2005). El desarrollo y la gestión de competencias profesionales: una mirada desde la formación. Revista Iberoamericana de Educación, 37(2), 1-15.

Valdez, J. L. (1998). Las redes semánticas naturales. Usos y aplicaciones en psicología social (4 ed.). México: Trillas.

Valencia, A. y Lizandra, J. (2018). Cambios en la representación social de la educación física en la formación inicial del profesorado. Retos, 34, 230-235.

Weisz, C. B. (2017). La representación social como categoría teórica y estrategia metodológica. Revista CES Psicología, 10(1), 99-108. https://doi.org/10.21615/cesp.10.1.7 